\title{
Multi-Purpose Robot using Raspberry Pi \& Controlled by Smartphone
}

\author{
K.S.R.Sastry ${ }^{1}$ (Asst.Professor), A.N.V Samba siva Rao ${ }^{2}$, K.Baji Babu ${ }^{3}$, \\ N.Rama Krishna ${ }^{4}$ \\ ${ }^{1}$ Dept. of Electronics and Communication Engineering., Andhra Loyola Institute of Engineering and \\ Technology., Vijayawada, India. \\ ${ }^{2}$ Dept. of Electronics and Communication Engineering., Andhra Loyola Institute of Engineering and \\ Technology., Vijayawada, India. \\ ${ }^{3}$ Dept. of Electronics and Communication Engineering, Andhra Loyola Institute of Engineering and Technology, \\ Vijayawada, India \\ ${ }^{4}$ Dept. of Electronics and Communication Engineering., Andhra Loyola Institute of Engineering and \\ Technology., Vijayawada, India.
}

\begin{abstract}
This paper proposes a method for controlling a Wireless robot for multi-purpose in the sense surveillance, rescue operations and weather reporting, human detection, geo-graphical location etc., Generally surveillance robots consists a high cost microcontroller, Bluetooth module or Wi-Fi modules, audio systems and a costly and complicated communication system. Raspberry pi b model is a credit card size single board computer. It is built with the Wi-Fi, Bluetooth modules and USB ports and in built Broadcom BCM2837 processor that gives very speed processing and audio, video jacks and camera interfacing port, HDMI port and Ethernet ports is available. This board will meet our requirements. The Robot is equipped with various critical sensors, detectors and Camera. The live video feedback is obtained from the camera. Data from the sensors is send to the Smartphone at user end. Latest Raspberry pi board is used on robot to interface with sensors and camera. It is having an in built Wi-Fi. An Android Smartphone sends a wireless command through robot controlled app which is received by Raspberry pi board and accordingly robot moves.
\end{abstract}

Keywords: Android, Bluetooth,Camera, Raspberry pi, Robot, Smartphone, Wi-Fi.

\section{INTRODUCTION}

Surveillance is the process in which people, places, or things are monitored usually for the purpose of influencing, managing, directing, or protecting them. Today there is a need for high surveillance especially in sensitive areas, military borders, government offices, public places, security check points and even in the homes. Country borders, public places, government offices etc., comes in the category of outdoor surveillance while warehouses, homes, garages fall in the category of indoor surveillance. Both these surveillance can include observation by means of CCTV (Closed Circuit Television) cameras or by sending soldiers or spies near the area which has to be examined. But CCTV and other cameras have their own drawbacks and constraints such as they usually have blind spots and they can be covered with sticking something over the lenses like gum or spray paint, also to cover the entire area many cameras are required to reach every nook and corner increasing the cost. Similarly human beings also have their own limitations such as obtaining information from inaccessible places is not possible; at the same time human life is precious and getting caught also a risk.
Thus humans cannot always be used to acquire information from remote and dangerous places. Here robots come into the picture. A robot capable of moving in all types of terrains and mounted with camera and other sensors can do the job. It can help in outdoor surveillance by monitoring important places and also it can be made to reach suspicious place for close observations. Similarly a robot can be used for indoor surveillance and can be made to reach the places of interest for getting the information rather than installing cameras in the whole environment [1]. With the use of the robots many human lives can be saved because an observation can be made without going to the risky places, also even if robot is caught it can be destroyed without risking anything.

Disasters can be classified as natural and man-made disasters. Natural disasters include cyclones, floods, storms, volcanic eruptions, earthquakes, Tsunami, hailstorms, wildfire, etc. Manmade disasters are nuclear power plant accidents, war, minefields, oil spills, radiation, hijacks, etc. Such disasters strike countries causing huge destruction and human deaths in the number of millions. During such situations to save lives of people numerous essential services are deployed. As 
reported by USAR (Urban Search and Rescue), the possibility of saving a victim is high within the first 48 hours of rescue operation after that probability becomes nearly zero [2].

Rescue involves responsive operations that involves saving the life of people stuck in a dangerous situation. In most of the cases rescue team is unable to enter in some difficult parts of affected area. Robotic veachile that moves in earthquake prone area and helps in identifying the live people. Even if the team is able to reach the people it is dangerous and risky to enter such areas without getting the prior information about the number of victims, their condition and environmental conditions of the area. Rescuing people from a hijack situation also requires a detailed information about the positions of hostages and terrorists so that the rescue members can prepare themselves. Sometimes due to lack of information a rescuer may become a victim [3]. In such risky and dangerous situations a robot could be a great help to the rescue team and can greatly improve the safety of the rescue team as well as their working efficiency [4]. A robot capable of moving in all types of terrain mounted with the camera and sensors can go to the affected area and can provide all the live information to the rescue team, as per the situation the rescue team can plan its activity.

The robot should be able to provide live video of the situation, detect human presence and other environmental related information such as humidity, temperature, presence of smoke, geographic location in terms of latitude and longitude. At the same time the user should be able to control and view information remotely.

\section{RELATED WORK}

Our concept is rather unique in the sense that it provides a low cost solution that offers unlimited range, video feedback. The robot is able to detect human presence. It is provided with temperature, humidity, smoke and gas sensors to measure various environment parameters.

The use of raspberry pi allows the use of many applications that can be helpful in surveillance and rescue operations. Acceleration and GPS data are received from sensors in robot. It can also detect humans if present in the vicinity. The robot can be controlled from a Laptop or Smartphone solving the problem of portability of controlling system.[5] Section II describes the related work Section III describes the proposed system. Section IV describes the system description Section V deals the sensors used in robot. VI. Related output samples finally conclude this paper in section VII.

\section{PROPOSED SYSTEM}

Our system includes a four wheel robot with a latest raspberry pi board and related hardware with camera. The robot is embedded with Passive Infrared, Temperature, Humidity, Smoke and GPS module. A Smartphone or Tablet is used to control the robot remotely over the Internet. Raspberry pi board controls the robot's motion, sensors, and other essential hardware (motors, chassis, power supply, etc.).[5] The robot connects to the Smartphone via raspberry pi's Wi-Fi module. The robot is provided with a battery to power Raspberry pi, Sensors and camera. It has also been provided with facility to charge battery when it is required. Fig. 1. Shows an overview of the proposed system.

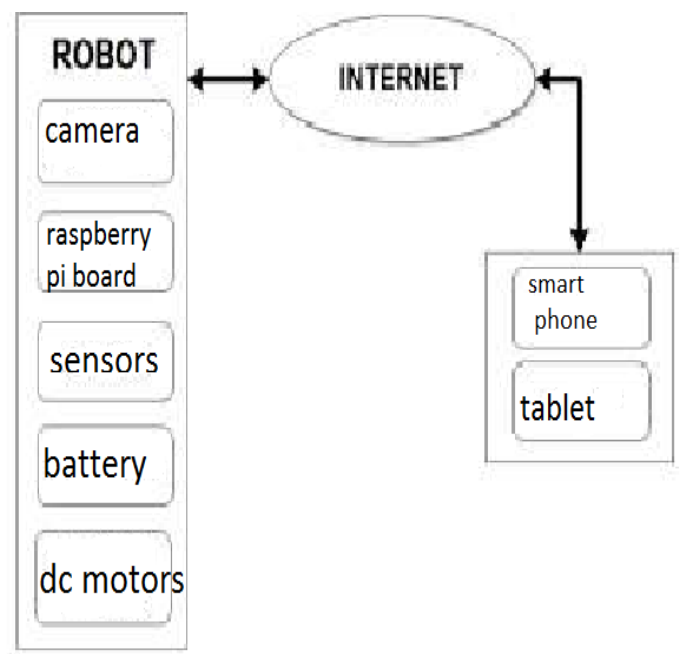

Fig. 1. Overview of System

Once the robot and sensors are ready, the raspberry pi board on the robot gets connected to Smartphone with Wi-Fi. The robot is remotely controlled by the Smartphone (with robot controlled application) or Tablet as per requirement. The live video and all the sensor data is sent to the user via internet. The user can navigate the robot easily with the help of live video feedback.

The control commands are sent to the robot using robot control app by Smartphone or Tablet. These commands are received by the raspberry pi which drives the robot in the required direction with the help of Motor Driver module.

The live video could be a great help in surveillance and rescue operations. Similarly the sensor readings like GPS position, magnetic field readings, quality of air, temperature and humidity values in the environment, can help the rescue personnel to prepare for the rescue mission. The Passive Infrared sensor can help to find the human trapped in the environment and thus personnel can rescue them easily without any extra work to find them and then reach them. 


\section{SYSTEM DESCRIPTION}

The system consists of a robot and a controlling device which could be a Laptop or a Tablet. A detailed block diagram is shown in Fig. 2.

The robot has four wheels and a uses a differential drive mechanism to move forward, backward and take left or right turn. The distance between the front and back wheel is kept minimum. This mechanism offers better traction and zero turning radius.

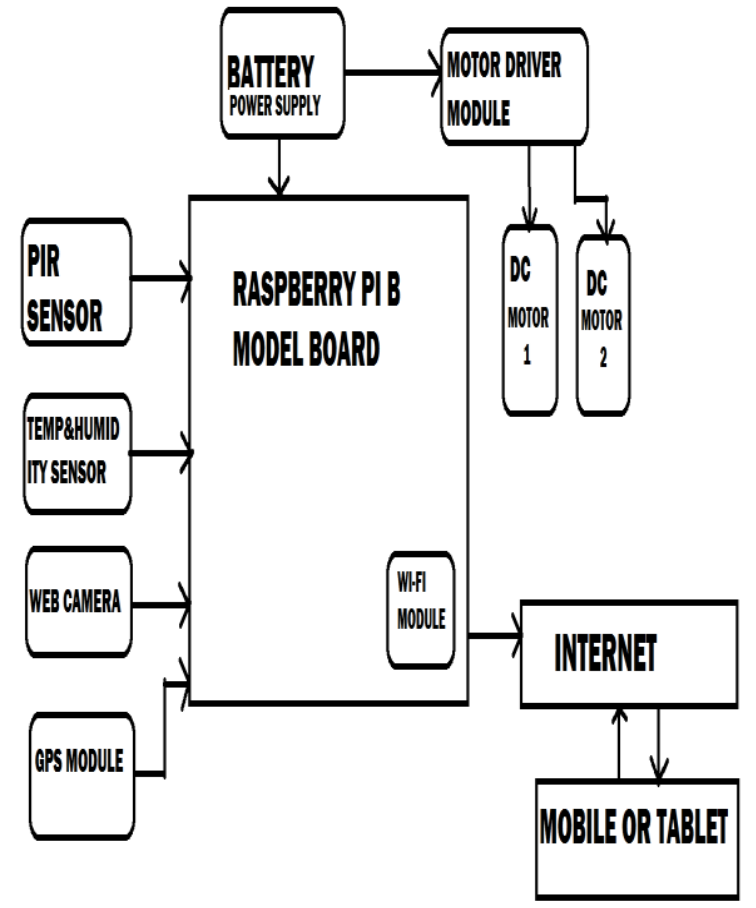

Fig. 2. A Complete Block Diagram

\section{A. power supply}

The primary requirement for our project is power supply. On the robot we can place a $12 \mathrm{v} 1 \mathrm{~A}$ battery. That is rechargeable. It provides the supply for the entire circuit. But the sensors required $5 \mathrm{v}$.for that we used a rectifier circuit. It converts $12 \mathrm{v}-5 \mathrm{v}$. Then that is supplied for sensors. Raspberry pi needs separate power bank because it requires up to $2.5 \mathrm{~A}$ current.

\section{B. The Raspberry pi 3 B model}

Raspberry $\mathrm{Pi}$ is used for making robot wireless and web based. Webcam is interfaced to the Raspberry Pi and then the videos are transmitted wirelessly from the robot to the user's monitor, from where the user can conveniently control the robotic vehicle's movement and also the data from sensors. Raspberry pi is connected with the Smartphone which enables raspberry pi to transmit over the web network.

Raspberry Pi uses an SD card for booting and for memory as it doesn't have an inbuilt hard disk for storage. Raspberry Pi requires 5 volt supply with minimum of 700-1000 mA current and it is powered through micro USB cable. ARM11 only requires 3.3 volt of supply which it takes with the help of linear regulator. 5 volt is required for the USB ports. It operates at $1.2 \mathrm{GHz}$ (Roughly $50 \%$ faster than $\mathrm{Pi} 2$ ) we use python to write the code into the raspberry pi. It has a strong processing capability due to the Broadcom BCM2837 processor \& Quadcore ARM Cortex-A53, 64Bit GPU ARM11 architecture and Linux-based system. In terms of interface and control, it has Video core IV Multimedia Co Processor, 802.11n wireless LAN (Wi-Fi) and Bluetooth 4.1, USB Ports, 4 x USB 2.0 GPIOs 2 x 20 Pin Header. 2x I2C, 2x SPI, 2x UART which basically meet the control requirement. There are easy to use open source peripheral driver libraries. [6]

\section{Android Smartphone}

An Android Smartphone with Bluetooth, Wi-Fi with Internet The Smartphone at user end will show the live video as well as the sensors output. With the help of robot controlled app we can access the robot and give the directions to robot e.g.: left, right, back etc...

\section{Motor Driver Module}

The L293D Motor Driver Module is used to drive the motors of the robot. The L293 is designed to provide bidirectional drive currents of up to $1 \mathrm{~A}$ at voltages from $4.5 \mathrm{~V}$ to $36 \mathrm{~V}$.

In L293D two h-bridges are present. Four transistors are present in each h-bridge. If we give logic bits 1,0 then current flow is $\mathrm{Vcc}$ to motor positive after that motor positive to negative and then flows to ground. Then motor rotates in one direction. We change the logic bits as 0,1 then current flow is Vcc to motor negative after that motor negative to positive and then flows to ground. Then motor rotate in opposite direction. If we give logic bits 1,1 then $\mathrm{Vcc}$ and ground are short. So motor does not rotate. If we give logic bits 0,0 then motor does not start. Because two pins are given to zero. [7]

\section{E. Dc motors}

The dc motors is used as wheels for our robot. These motors required $12 \mathrm{v}, 600 \mathrm{MA}$. Which is provided by $\mathrm{L} 293 \mathrm{~d}$ ic and then it will rotate at 45 RPM.

\section{F. Web camera}

The visual feedback is provided by the Intex IT-306WC webcam. It can have a resolution of up to 30.0MP, Frame rate of 30FPS along with night time vision. It is plugged into the USB port of the Raspberry Pi. It gives us the video feedback.

According to that we can move our robot in desired direction. 


\section{SENSORS USEDIN ROBOT}

The following sensors are embedded in the robot that could be useful in surveillance and rescue operations.

\section{A. Passive Infrared Sensor}

It is passive infrared sensor which detects the motion with the variation of infrared radiation. It consists of multiple facets with each part containing Fresnel lens. Fresnel lens condenses light providing a large range of IR (Infrared) to the sensor. It provides single bit digital output and is compatible with all microcontrollers. The motion of injured person can be detected with this sensor [8].

\section{B. The Temperature And Humidity Sensor Module \\ DHT11 Temperature \& Humidity Sensor} features a temperature \& humidity sensor complex with a calibrated digital signal output. By using the exclusive digital-signal acquisition technique and temperature \& humidity sensing technology, it ensures high reliability and excellent long-term stability. This sensor includes a resistive-type humidity measurement component and NTC temperature measurement component, which connects to a raspberry pi GPIO pins, offering excellent quality, fast response, anti-interference ability and cost-effectiveness [9]. This sensor is used in robot for measuring temperature and humidity during rescue operation.

\section{C.GPS module}

It is used to navigate our robot at forests, deserts, sensitive places. It is interface with raspberry pi.

\section{RELATED OUTPUTS}

Transmission of Video via Wi-Fi to a Smartphone

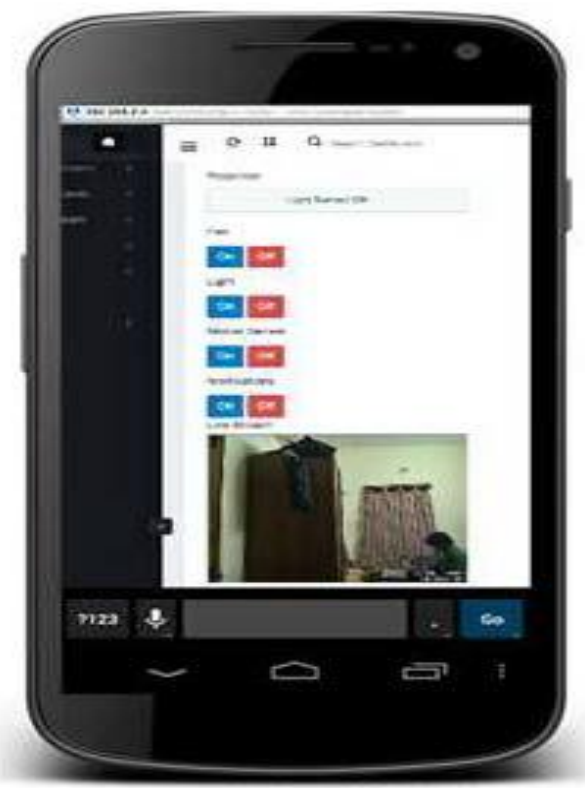

Fig 1:- Final Video on mobile with robot control app

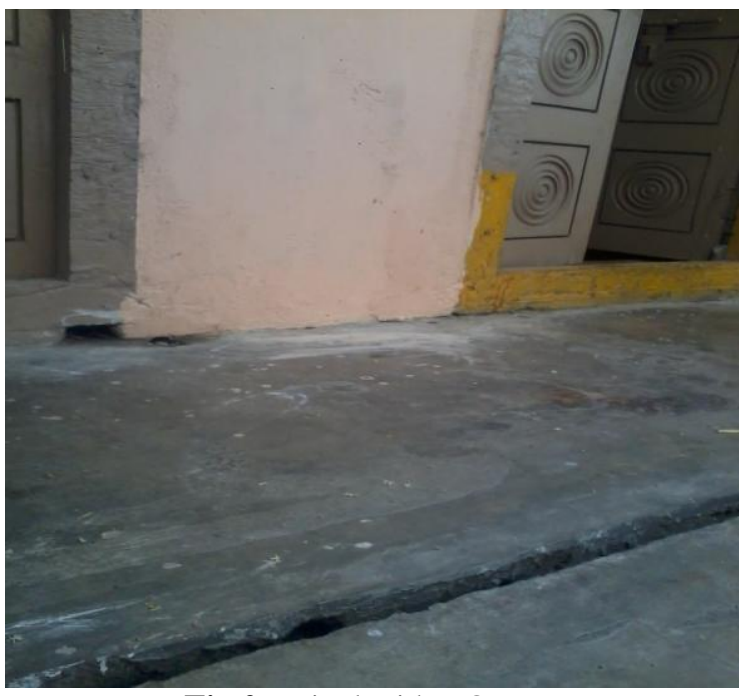

Fig 2:- Final Video Output

The above fig. 1 shows how the mobile at user end and it displays the live video streaming with robot control commands. The fig. 2 shows the full video output captured by webcam.

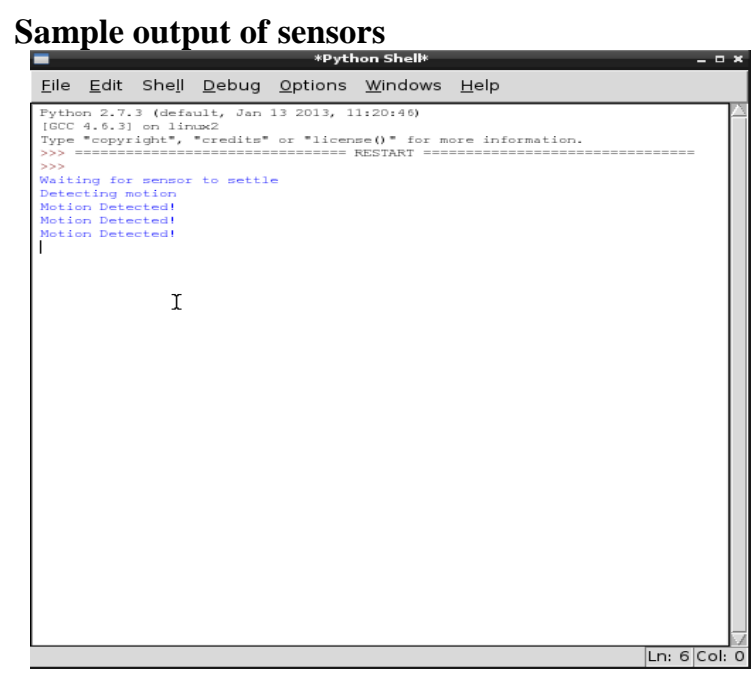

Fig 3:- Pir sensor output

\begin{tabular}{|c|c|c|c|}
\hline & \multicolumn{1}{|c|}{ A } & \multicolumn{2}{c|}{ B } \\
\hline 1 & Date & Temp & Humidity \\
\hline 2 & Saturday, September 27, 2014 at 8:53:59 PM & 21.70 & 59.60 \\
\hline 3 & Saturday, September 27, 2014 at 8:54:31 PM & 21.60 & 59.60 \\
\hline 4 & Saturday, September 27, 2014 at 8:55:03 PM & 21.60 & 58.90 \\
\hline 5 & Saturday, September 27, 2014 at 8:55:35 PM & 21.60 & 59.10 \\
\hline 6 & Saturday, September 27, 2014 at 8:56:07 PM & 21.60 & 62.00 \\
\hline 7 & Saturday, September 27, 2014 at 8:56:39 PM & 21.60 & 62.90 \\
\hline 8 & Saturday, September 27, 2014 at 8:57:11 PM & 21.60 & 62.70 \\
\hline 9 & Saturday, September 27, 2014 at 8:57:43 PM & 21.70 & 62.70 \\
\hline 10 & Saturday, September 27, 2014 at 8:58:15 PM & 21.70 & 62.10 \\
\hline 11 & Saturday, September 27, 2014 at 8:58:50 PM & 21.60 & 61.60 \\
\hline 12 & Saturday, September 27, 2014 at 8:59:22 PM & 21.60 & 61.70 \\
\hline 13 & Saturday, September 27, 2014 at 8:59:54 PM & 21.60 & 62.50 \\
\hline 14 & Saturday, September 27, 2014 at 9:00:26 PM & 21.70 & 62.50 \\
\hline
\end{tabular}

Fig 4:- DTH sensor sample output

The fig.3 shows the output of PIR sensor motion detection and fig. 4 shows the DTH sensor output 


\section{Software Required}

Python language used for writing program on raspberry pi. Jessie (OS) for raspberry pi installation.

\section{CONCLUSION}

The Raspberry $\mathrm{Pi}$ is used to control robot using Smartphone from remote location. The time required for processing the commands from the smart phone and responding accordingly is negligible. The experimental result has proved that the fetching of a good quality video is quick and clear. The use of Smartphone instead of Computer and Laptop make the system more reliable and easy to use.

It can also measure the temperature and humidity values and can detect harmful gases if present. The robot can be easily controlled remotely with the help of a smartphone or a Tablet over the Internet. For indoor use, it can detect gas leak in home or industry, surveillance of shopping mall, parking lot, office, industry, bank, museums, rescuing operation during natural disaster and aftermath like searching for human, supplying food and other essentials to the people under unreachable ruin. It can also perform military surveillance like spying enemy base, exploring unknown enemy territory, and as communication device during war, natural disasters.

\section{FUTURE SCOPE}

Raspberry pi latest model offers us so many features like HDMI port,GPU and offers 4 USB ports and 40 GPIO pins we should make use of it and try to build a very powerful and intelligent robot. Power supply is also a major constraint for any type of robot so solar panel may meet our requirement but it is bulk in size.

\section{ACKNOWLEDGMENT}

The authors gratefully acknowledge the support from M.Rama Krishna (Head of Department ECE, ALIET), CH. Pranob Kumar (projectcoordinator for $2^{\text {nd }}$ division ECE ALIET), All teaching staff from ECE-II (ALIET) for their support who are initiated and provided require facilities to do research work.

\section{REFERENCES}

[1]. Donato Di Paola, Annalisa Milella, Grazia Cicirelli and Arcangelo Distante, "An autonomous mobile Robotic system for surveillance of indoor environments", International Journal of Advanced Robotic Systems, Vol. 7, No. 1 (2010).

[2]. P. Vanitha Sri, S. Sharmila, K. Karthik, "A surveillance Robot for home security with docking system", in International Journal of Science, Engineering and Technology Research (IJSETR) on, Volume 4, Issue 11, November 2015.
[3]. Trupti B. Bhondve, Prof. R. Satyanarayan, Prof. Moresh Mukhedkar, "Mobile rescue Robot for Human body detection in rescue operation of disaster", in International Journal of Advanced Research in Electrical, Electronics and Instrumentation Engineering on, Vol. 3, Issue 6, June 2014.

[4]. Maki K. Habib, Yvan Baudoin, "RobotAssisted risky intervention search, rescue and environmental surveillance", in International Journal of Advanced Robotic Systems on, Vol. 7, No.1, ISSN 1729-8806, pp. 098, March 2010.

[5]. International Conference on Communication and Signal Processing, April 6-8, 2016, India by Mohammad Shoeb Shah and P. B. Borole

[6]. http://www.seeedstudio.com/wiki/Raspberry _Pi_3_Model_B

[7]. http://www.L293D/wiki/ full data sheet.

[8]. International Journal Of Business Intelligents Volume 02, Issue 01, June 2013

[9]. Dht11 temperature and sensor module [Online] Available: http://www.uugear.com/ portfolio/dht11-humidity temperature-sensor module. 